\title{
Anwendungsgrenzen, Behördenkooperation und Ermessensausübung des Rates im Rahmen der europäischen Terrorismusbekämpfung
}

\author{
- Anmerkung zum Urteil des EuG vom 16.10.2014, \\ verb. Rs. T-208/11 und T-508/11 \\ (Liberation Tigers of Eelam/Rat der Europäischen Union) -
}

\author{
Von Ferdinand Weber, Göttingen*
}

1. Ein bewaffneter Konflikt im Sinne des humanitären Völkerrechts schließt die Anwendbarkeit unionsrechtlicher Terrorismusvorschriften nicht aufgrund Spezialität aus. Ihre Anwendbarkeit richtet sich allein nach unionsrechtlichen Determinanten.

2. Die Union darf sich für die Begründung der Aufnahme natürlicher und juristischer Personen in den Anhang einer ,smart sanctions"-Verordnung auf Behördeninformationen aus Drittstaaten stützen, soweit verfahrens- und menschenrechtliche Mindeststandards im informationsgebenden Staat gewahrt werden.

3. Dem Rat steht bei der Listenerstellung ein weites Ermessen zu, dessen Ausübung jedoch vom Unionsrichter überprüfbar ist. Dabei ist der Rat strikt an die Einhaltung des vom Sekundärrecht vorgesehenen zweistufigen Informations- und Entscheidungssystems gebunden.

Urteil des EuG vom 16. Oktober 2014, verb. Rs. T-208/11 und T-508/11 - Leitsätze des Verfassers

Die gegen Individuen gerichteten Terrorismusbekämpfungsnormen der Europäischen Union sind seit ihrer Entstehung ein Gebiet, in dem die Unionsgerichtsbarkeit in der Pflicht steht, einen Ausgleich zwischen Sicherheitsinteressen und grundlegenden Rechten des Einzelnen zu finden. Den Unionsgerichten stehen offen formulierte Rechtsgrundlagen und mit Kommission und Rat zwei selbstbewusste Organe gegenüber, die ein weitgehendes Ermessen im Rahmen der Entscheidung über ,,smart sanctions" reklamieren. Dem mussten die Gerichte wiederholt entgegentreten und nach der Anwendbarkeit der Unionsgrundrechte auch grundlegende Verfahrenspflichten der Organe herausarbeiten. Dabei treten noch immer Fragen des Verhältnisses zum Völkerrecht und eigenwillige Auffassungen von Verfahrensstandards zu Tage.

\section{Die Zweigleisigkeit des unionsrechtlichen smart-sanction-Regimes}

Die unionsrechtlichen Normen zur Terrorismusbekämpfung dienen dem völkerrechtlichen Vorhaben der Staatengemeinschaft, terroristische Aktivitäten einzu-

* Dipl.-Jur. Ferdinand Weber, MLE. ist wiss. Mitarbeiter am Lehrstuhl für Öffentliches Recht und Europarecht am Institut für Völkerrecht und Europarecht der Georg-August-Universität Göttingen. Der Verfasser möchte Prof. Dr. Frank Schorkopf und PD Dr. Marcus Schladebach für hilfreiche Anmerkungen danken. 
dämmen und bauen auf staatliche Unterstützung und Umsetzung. Der Rechtsbereich wurde seit der Jahrtausendwende auf Unionsebene richterrechtlich ausgeformt und nur teilweise positivrechtlich nachvollzogen. Im Rahmen dieses Kooperationsverbundes hatte das EuG nun auf neue Fragen zu antworten: Unterliegt das unionsrechtliche smart-sanction-Regime im Rahmen bewaffneter Konflikte einer völkerrechtlichen Anwendungssperre? Darf der Rat im Rahmen der Terrorismusbekämpfung mit Behörden von Drittstaaten kooperieren, wenn deren rechtsstaatliche Standards von jenen der Union abweichen? Das geschriebene Unionsrecht gibt hierauf keine eindeutigen Antworten. Darüber hinaus ist dem EuG hier Gelegenheit gegeben, die Begründungspflicht des Rates - wie jene der Kommission im zweiten Kadi-Verfahren ${ }^{1}$ - zu präzisieren. Dabei ruht der unionsrechtliche Rechtskomplex auf zwei zu unterscheidenden sekundärrechtlichen Grundlagen, zu denen sich zwei Rechtsprechungslinien entwickelten.

Die Union bemüht sich seit der auf Sicherheitsratsebene eingeleiteten Terrorismusbekämpfung um eine einheitliche Umsetzung des zuerst gegen die Taliban, bald auf weitere natürliche und juristische Personen ausgeweiteten Vorhabens, Vermögenswerte der Betroffenen bis auf das existenziell Notwendige einzufrieren. ${ }^{2}$ Dabei erstellt ein vom Sicherheitsrat eingesetzter Sanktionsausschuss auf Grundlage staatlicher Behörden- und Geheimdienstinformationen Namenslisten. Die Staatengemeinschaft wurde jedoch zusätzlich aufgerufen, selbst unmittelbar tätig zu werden. ${ }^{3}$ Aufgrund der bis zum Änderungsvertrag von Lissabon bestehenden Kompetenzausstattung und -verteilung wurden die im Rat der Europäischen Union vereinigten Vertreter der Mitgliedstaaten im Rahmen der GASP nach Art. 15 und 34 EUV a.F. tätig und erließen einen inhaltlich an die einschlägigen Sicherheitsratsresolutionen anknüpfenden Gemeinsamen Standpunkt. Im Anschluss konnten die Organe der Europäischen Gemeinschaft auf Grundlage der Brückenklausel des Art. 301 i.V.m. Art. 308 und 60 EGV Verordnungen erlassen. Dabei schuf die Union zwei sekundärrechtliche Sanktionsregime: durch eine Grundverordnung erlangt die auf Sicherheitsratsebene erstellte Liste unmittelbare Geltung in allen Mitgliedstaaten. ${ }^{4}$ Die Namenslisten des Sicherheitsrats werden hierbei schlicht übernommen und die Kommission nur dazu ermächtigt, die Liste gemäß den Änderungen auf völkerrechtlicher Ebene zu aktualisieren. Ein Durchführungsermessen steht ihr nicht zu. Mit einer weiteren Grundverordnung verhängt die Union darüber hinaus Sanktionen auf Grundlage einer vom Rat selbst erstellten, auf staatlichen Behördeninformationen beruhenden Liste und begründet

1 Hierzu N. Weiß, Praktische Konsequenzen der Kadi-Rechtsprechung, EuR 2014, S. 234 f. m.w.N.

2 Vgl. Ziff. 4 (b) S/RES/1267(1999); Ziff. 8 (c) i.V.m. 16 (b) S/RES/1333(2000); Ziff. 1 (c) S/RES/1373(2001); Ziff. 1 und 2 (a) S/RES/1390(2002) sowie Ziff. 1 (a) S/RES/1989(2011).

3 Ziff. 1 (c) S/RES/1373(2001) war insoweit eine direkte Reaktion auf die Anschläge vom 11. September 2001.

4 Gemeinsame Standpunkte 1999/727/GASP, 2001/154/GASP, 2002/402/GASP; seit dem Lissabonner Vertrag nach Art. 29 EUV als Beschluss: Beschluss 2011/487/GASP; daran anknüpfend und seit Lissabon gem. Art. 215 II, 352 und 60 AEUV die Verordnungen (EG) Nr. 337/2000, 467/2001, 881/2002, zuletzt aktualisiert durch die Durchführungsverordnung (EU) Nr. 1193/2014 der Kommission vom 4. November 2014. 
so ein zweites Sanktionsregime in vollständiger unionsrechtlicher Eigenverantwortung. 5

Parallel zum zweigleisigen Sanktionsregime der Union entwickelten sich zwei Rechtsprechungslinien zu den eingehenden Nichtigkeitsklagen Betroffener. Sie sorgten auch im Gerichtssystem der Union angesichts der zu klärenden Grundsatzfragen für Konflikte. Das EuG ist nach Art. 256 Abs. 1 S. 1 AEUV i.V.m. Art. 263 Abs. 4 AEUV für Klagen Betroffener zuständig, weshalb im Rahmen der Terrorismusbekämpfung ein umfassendes Verständnis der Rechtsprechungsentwicklung ohne Berücksichtigung der erstinstanzlichen Judikate unvollständig bleibt. Hinsichtlich der völkerrechtlich determinierten Liste stellt sich die Frage nach dem Verhältnis des Unionsrechts zum Völkerrecht vor dem Hintergrund des Ausgleichs zwischen dem Funktionieren des völkerrechtlichen Friedenssicherungssystems und effektivem Grundrechtsschutz („Kadi“-Rechtsprechung) ${ }^{6}$. Bemerkenswert ist, dass das EuG dem EuGH im Ergebnis mehr aufgrund des Instanzenzugs denn aus Überzeugung folgt: im Urteil zur zweiten Nichtigkeitsklage des Herrn Kadi betont es, im konkreten Fall zwar nicht an die rechtliche Beurteilung des Gerichtshofs gebunden zu sein, dass es jedoch aufgrund der hierarchischen Struktur der Gerichte ratsam erscheint ,die in der Entscheidung des Gerichtshofs (Kadi I, d. Verf.) vorgenommene rechtliche Beurteilung nicht selbst in Frage zu stellen" 7 . Der EuGH hielt im zweiten Verfahren dennoch an der vollen Justiziabilität der völkerrechtlich induzierten Unionsrechtsakte fest, präzisierte seine Vorgaben zur grundrechtlich veranlassten Begründungspflicht der Kommission und machte Angaben zum Umfang der gerichtlichen Kontrolldichte. ${ }^{8}$

Institutionell und grundrechtlich ebenso bedeutend gestalteten sich die Problemstellungen bezüglich der in Eigenverantwortung des Rates erstellten Terrorliste. Hier konnte der EuGH vom EuG mit kompetenziellen Argumenten belassene Rechtsschutzlücken gegenüber intergouvernementalen Unionsrechtsakten ${ }^{9}$ nur mit einer teleologisch begründeten Überwindung deutlicher Wortlautgrenzen vermeiden. ${ }^{10}$ Hinsichtlich der die „Unionsliste“ umsetzenden Verordnungen stellten sich aber immerhin keine völkerrechtlichen Probleme, sodass die Unionsgerichts-

5 Gemeinsamer Standpunkt 2001/931/GASP, fortlaufend aktualisiert durch Beschlüsse, zuletzt 2014/483/ GASP; daran anknüpfend VO (EG) Nr. 2580/2001 des Rates, zuletzt aktualisiert durch Durchführungsverordnung (EU) Nr. 790/2014 des Rates vom 22. Juli 2014.

6 In diese Rechtsprechungslinie gehören u.a. die Urteile des EuGH in den verb. Rs. C-402/05 P und C-415/05 P (Kadi I), Slg. 2008 I-2351; Rs. C-399/06 und C-403/06 (Ayadi und Hassan), Slg. 2009 I-11393; Rs. C-584/10 P, C-593/10 P und C-595/10 P (Kadi II), bisher nicht in der Sammlung veröffentlicht.

7 Vgl. hierzu EuG, Rs. T-85/09 (Kadi II), Slg. 2010 II-5177, Rn. 112, 115, 121.

8 Für Einzelheiten vgl. $N$. Weiß (Fn. 1), S. 231 ff.

9 Exemplarisch der klageabweisende Beschluss des EuG, Rs. T-338/02 (Segi u.a.), Slg. 2004 II-1647, Rn. 38: „Zum von den Klägern geltend gemachten Fehlen eines wirksamen Rechtsbehelfs ist festzustellen, dass die Kläger wahrscheinlich über keinen wirksamen gerichtlichen Rechtsbehelf vor den Gemeinschaftsgerichten oder den nationalen Gerichten gegen die Aufnahme von Segi in die Liste von Personen, Vereinigungen oder Körperschaften, die an terroristischen Handlungen beteiligt sind, verfügen.".

10 Da Gemeinsame Standpunkte normalerweise keine Drittwirkung entfalten, sei eine Prüfung wegen Art. 6 II EUV a.F. entgegen dem Wortlaut des Art. 35 I EUV a.F. angezeigt, vgl. EuGH, Rs. C-355/04 P (Segi u.a.), Slg. 2007 I-1657, Rn. 52 f.; inzwischen ist die Unionsgerichtsbarkeit auch formal zur umfassenden Gewährung von Rechtsschutz befugt, vgl. Art. 275 AEUV. 
barkeit problemlos eine Grundrechtsbindung des Rates annehmen konnte. ${ }^{11}$ Damit lag das Augenmerk der die Unionsliste betreffenden Klagen von Beginn an auf dem Umfang der Begründungspflicht des Rates gegenüber Betroffenen im Rahmen der Listenerstellung und -aktualisierung ${ }^{12}$ sowie den Anforderungen an Beschlüsse informationsgebender staatlicher Behörden im Sinne des Sekundärrechts. ${ }^{13}$

Der zu besprechende Fall fällt unter das zuletzt beschriebene Sanktionsregime. Für die vom Rat selbst erstellte Liste ist dabei vom Gemeinsamen Standpunkt 931/2001/GASP ein zweistufiges Informations- und Entscheidungssystem mit strikter Aufgabentrennung vorgesehen: nach Art. 1 Abs. 4 erstellt der Rat die Liste selbst, muss sich hierbei aber zur Begründung gegenüber Betroffenen auf genaue Informationen sowie ernsthafte und schlüssige Beweise oder Indizien von für Terrorismusbekämpfung zuständigen staatlichen Behörden stützen. Dies sind in der Regel Justizbehörden. Der Rat trifft damit auf Unionsebene eine Ermessensentscheidung, die jedoch auf konkreten staatlichen Ermittlungen oder Verurteilungen beruhen muss. Die Liste ist gem. Art. 1 Abs. 6 des Standpunkts regelmäßig zu aktualisieren, um sicherzustellen, dass der Verbleib der Betroffenen auf ihr nach wie vor gerechtfertigt ist. Diese im Rahmen der GASP erstellte Liste wird aufgrund Art. 2 Abs. 3 VO (EG) Nr. 2580/2001 Bestandteil der Grundverordnung. Die Klägerin des zu besprechenden Falles wendet sich gegen ihre 2006 erfolgte und seitdem anhaltende Listenaufnahme durch den Rat.

\section{Hintergrund der Entscheidung und aufgeworfene Rechtsfragen}

Die Klägerin kämpfte seit Jahrzehnten mit militärischen Mitteln für eine staatliche Unabhängigkeit der Tamilen im Norden und Osten Sri Lankas und übte zeitweise die alleinige Gewalt in weiträumigen Gebieten aus. Nach gescheiterten Friedensverhandlungen mit der Regierung im Jahr 2006 und erneut zunehmender Gewalt setzte der Rat sie auf die o.g. Liste. 2009 erlitt die Klägerin eine vollständige militärische Niederlage, besteht jedoch fort ${ }^{14}$ und begehrt die Nichtigerklärung der sie auf die Liste setzenden Durchführungsverordnungen sowie die Feststellung, dass die o.g. Grundverordnung auf sie keine Anwendung findet.

Dafür führt sie sieben Klagegründe an, die vom EuG zu drei Klagegründen zusammengefasst ${ }^{15}$ und entsprechend gemeinsam behandelt werden: Erstens be-

11 So bereits das EuG, Rs. T-228/02 (Volksmudschaheddin des Iran I), Slg. 2006 II-4665, Rn. 101 ff., 137 ff.

12 Vgl. EuG, Rs. T-284/08 (Volksmudschaheddin des Iran II), Slg. 2008 II-3487, Rn. 36 f., 55, 73 ff.; bestätigt durch EuGH, Rs. C-27/09 P (Frankreich/People's Mojahedin Organization of Iran), Slg. 2011 II-13472, Rn. 62 f., 66.

13 Hierzu EuG, Rs. T-341/07 (Sison), Slg. 2009 II-3625, Rn. 111 f.; EuGH, Rs. C-539/10 P (Al-Aqsa), veröffentlicht in der digitalen Sammlung (Allgemeine Sammlung), Rn. $64 \mathrm{ff}$.

$14 \mathrm{Zu}$ neuerer politischer Instabilität und nicht aufgeklärten Verbrechen der Armee an der tamilischen Zivilbevölkerung vgl. F.A.Z. vom 5. Januar 2015, S. 5, Unter einem schlechten Stern sowie F.A.Z. vom 10. Januar 2015, S. 7, Das Ende des Triumphalismus.

15 EuG, verb. Rs. T-208/11 und T-508/11 (Liberation Tigers of Eelam/Rat der EU), noch nicht in der Sammlung veröffentlicht, Rn. 40 ff., 153 ff. 
hauptet die Klägerin die Unanwendbarkeit der unionsrechtlichen Terrorismusvorschriften aufgrund der Spezialität humanitären Völkerrechts. Bei dem bewaffneten Konflikt zwischen ihr und der Regierung handle es sich um einen Selbstbestimmungskampf des tamilischen Volkes, womit ihr Kombattantenstatus zukomme. Damit aber sei die Anwendung der Grundverordnung ausgeschlossen, andernfalls würde ihre kriegsvölkerrechtliche Immunität verletzt. Dies gelte auch für unrechtmäßige Handlungen, deren Behandlung sich allein nach humanitärem Völkerrecht bemesse. Andernfalls würde die Union nach humanitärem Völkerrecht möglicherweise entschuldbare Handlungen durch die Terrorismusklassifizierung kriminalisieren. Damit sei die Listenaufnahme als Einmischung eines Drittstaats in einen bewaffneten Konflikt anzusehen und somit der Grundsatz der Nichteinmischung verletzt. Die Klägerin fügt ein unionsrechtliches Argument hinzu, indem sie auf Art. 1 Abs. 3 931/2001/GASP verweist, nach dem Handlungen, die nach innerstaatlichem Recht keine Straftat darstellen, nicht unter den Gemeinsamen Standpunkt fallen. Da rechtmäßige Kriegshandlungen nicht als rechtswidrig angesehen werden können, sei das Unionsrecht nicht nur verdrängt, auch sein Anwendungsbereich wäre konkret nicht eröffnet. ${ }^{16}$ Zweitens seien die im konkreten Fall informationsgebenden britischen und indischen Behörden, auf deren Erkenntnisse der Rat im Rahmen seiner Begründung zur Belassung der Klägerin auf der Liste Bezug nimmt, keine zuständigen Behörden, wie sie Art. 1 Abs. 4 des Gemeinsamen Standpunkts definiert. Im Vereinigten Königreich gebe es zuständige Justizbehörden im Sinne des Gemeinsamen Standpunkts, vorliegend wurden jedoch Verwaltungsbehörden tätig und stuften die Klägerin als terroristische Vereinigung ein. Da es an Strafverfolgungsmaßnahmen oder der Aufnahme von Ermittlungen durch eine Justizbehörde fehle, seien die Vorgaben des Art. 1 Abs. 4 nicht gewahrt. ${ }^{17}$ Indische Behörden könnten aufgrund des Grundsatzes der loyalen Zusammenarbeit aus Art. 4 Abs. 3 EUV von vornherein keine zuständigen Behörden sein, die Vorschrift beziehe nur mitgliedstaatliche Behörden ein. Der Rat könnte sich sonst auf Informationen aus Drittstaaten stützen, ohne dass, wie innerhalb der Union, die Wahrung der Verteidigungsrechte der Betroffenen sichergestellt sei. Im Übrigen gelte das zu den britischen Behörden Vorgetragene. ${ }^{18}$ Drittens macht die Klägerin geltend, der Rat habe sich im Rahmen der Überprüfung nach Art. 1 Abs. 6 des Gemeinsamen Standpunkts, ob der Verbleib der Klägerin auf der Liste weiterhin gerechtfertigt ist, zur Begründung seiner Entscheidung im Wesentlichen nicht auf Behördeninformationen gestützt, sondern auf eine selbst zusammengestellte Liste von Handlungen, die er ihr unmittelbar zur Last legt. Aufgrund dieser einer rechtlichen und tatsächlichen Grundlage entbehrenden Handlungszurechnung im Rahmen der Begründung für den Listenver- 
bleib sei auch eine Verteidigung nicht möglich gewesen und somit ihre Verteidigungsrechte verletzt. ${ }^{19}$

\section{Die Entscheidung des EuG}

Das EuG erklärt die Durchführungsverordnungen, die die Klägerin auf der Liste der Grundverordnung belassen, für nichtig, soweit sie diese betreffen. Der Rat durfte sich im Ergebnis nicht auf die Angaben der indischen Behörden verlassen und verletzte darüber hinaus die ihm obliegende Begründungspflicht. Die übrigen Klagegründe weist es zurück, bringt jedoch einige Klarstellungen hinsichtlich des die Unionsliste betreffenden Sanktionsregimes.

Dem ersten Klagegrund tritt das EuG mit unionsrechtlichen und völkerrechtlichen Argumenten entgegen: demnach können dem humanitären Völkerrecht unterfallende Handlungen die Anwendung unionsrechtlicher Vorschriften grundsätzlich nicht ausschließen, da sich ihr Anwendungsbereich allein nach dem Unionsrecht selbst richte. Dieses sieht hier jedoch nicht nur keine Ausnahmen vor, vielmehr ergingen der einschlägige Gemeinsame Standpunkt sowie die Grundverordnung gerade, um die Resolution 1373 (2001) des Sicherheitsrats umzusetzen. ${ }^{20}$ Mit dieser selbstreferentiellen, dualistisch geprägten Argumentation schließt das EuG insoweit an den EuGH in der Kadi-Rechtsprechung an, als es die Autonomie des Unionsrechts hervorhebt und dabei völkerrechtliche Erwägungen auf der Ebene von handlungsleitenden Motiven ansiedelt. Zur völkerrechtlichen Ebene stellt das Gericht im Anschluss fest, dass das humanitäre Völkerrecht selbst seinen Regeln zuwiderlaufende Handlungen als terroristisch einstufe und verbiete. Hierbei beruft es sich insbesondere auf Normen des Genfer Abkommens vom 12. August 1949 über den Schutz von Zivilpersonen in Kriegszeiten sowie dessen Zusatzprotokolle über den Schutz der Opfer internationaler und nicht internationaler Konflikte. Weiterhin weist es auf das Internationale Übereinkommen zur Bekämpfung der Finanzierung des Terrorismus vom 9. Dezember 1999 hin, das zeige, dass auch auf völkerrechtlicher Ebene eine parallele Anwendung humanitären Völkerrechts und völkerrechtlicher Terrorismusvorschriften geregelt sei. ${ }^{21}$ Der Grundsatz der Nichteinmischung gelte ohnehin nur für Staaten, weshalb er nicht anwendbar sei und der Hinweis der Klägerin auf Art. 1 Abs. 3 des Gemeinsamen Standpunkts gehe fehl, da dieser zwar für rechtmäßige Kriegshandlungen gelte, nicht jedoch für etwaige terroristische Handlungen, die im Rahmen eines bewaff-

$19 \operatorname{EuG}($ Fn. 15), Rn. 155.

20 EuG (Fn. 15), Rn. 57 ff.; vgl. die Erwägungsgründe (2) und (5) des Gemeinsamen Standpunktes 931/2001/ GASP sowie die Erwägungsgründe (3) und (5) der Verordnung (EG) Nr. 2580/2001 des Rates, die direkten Bezug auf S/RES/1373 (2001) nehmen.

21 Vgl. zur völkerrechtlichen Argumentation EuG (Fn. 15), Rn. 56 ff., unter Verweis auf Art. 2 Abs. 1 lit. b) des zuletzt genannten Übereinkommens. 
neten Konflikts begangen werden und damit zugleich unrechtmäßige Kriegshandlungen darstellen. ${ }^{22}$ Auf dieser Grundlage weist es den ersten Klagegrund zurück. Der zweite Klagegrund hat hingegen teilweise Erfolg. Aus der Auslegung des Art. 1 Abs. 4 UAbs. 2 931/2001/GASP ergebe sich zwar, dass eine Präferenz für Justizbehörden bestehe. Doch könnten auch andere Behörden beteiligt werden, soweit diese in auf Terrorismusbekämpfung ausgerichtete Verfahren eingebunden sind und restriktive Beschlüsse erlassen können. Damit knüpft das EuG an eine bereits seit der Rs. Al-Aqsa etablierte, zweckmäßigkeitsorientierte Auslegung der Vorschrift an. ${ }^{23}$ Auch die konkret beteiligten Behörden des Vereinigten Königreichs, das Home Secretary und das UK Treasury, fallen unter die Vorschrift. ${ }^{24}$ Fraglich blieb damit die Kooperation des Rates mit Behörden aus Drittstaaten, ${ }^{25}$ im vorliegenden Fall indischen Behörden. Der Grundsatz der loyalen Zusammenarbeit aus Art. 4 Abs. 3 EUV ist nach dem EuG jedenfalls kein Hinderungsgrund, da er schlicht nur Verpflichtungen zwischen Rat und mitgliedstaatlichen Behörden begründet. Daraus lässt sich jedoch noch nicht das Argument gewinnen, das eine über das Unionsgebiet hinausgehende Kooperation ausgeschlossen sei. Vielmehr sieht der Gemeinsame Standpunkt hier gerade keine Begrenzung auf mitgliedstaatliche Behörden vor und ergeht in Umsetzung o.g. Sicherheitsratsresolution, die die gesamte Staatengemeinschaft zur Kooperation aufruft. Damit können Drittstaatsbehörden grundsätzlich in das Entscheidungs- und Informationssystem zur Listenerstellung eingebunden werden. ${ }^{26}$ Jedoch muss der Rat im Rahmen jeder Drittstaatskooperation sorgfältig prüfen, ob die Verfahrensgrundrechte - gemeint sind die Verteidigungsrechte und das Recht auf einen effektiven gerichtlichen Rechtsschutz, wie sie für die Union in Art. 41 Abs. 2 und 47 GRC sowie Art. 6 EMRK niedergelegt sind ${ }^{27}$ - im jeweiligen Staat theoretisch und praktisch gewahrt werden, da dort weder die Grundrechtecharta noch möglicherweise die EMRK gelten. Diese Prüfung ist laut EuG Bestandteil der dem Rat obliegenden Begründungspflicht. Ein pauschaler Hinweis auf drittstaatliche Rechtsvorschriften und dort vorgesehene Kontrollmechanismen genügt nicht. ${ }^{28}$ Eine solche nicht näher spezifizierte - sorgfältige Prüfung hat der Rat vorliegend versäumt, erstmalige Hinweise vor Gericht können die Verletzung der Begründungspflicht

$22 \operatorname{EuG}($ Fn. 15), Rn. 69, 79 ff.

23 EuG (Fn. 15), Rn. 105, 107 unter Verweis auf EuG, Rs. T-348/07 (Al Aqsa), Slg. 2010 II-4575, Rn. 88 f. und EuGH, Rs. C-539/10 P (Al-Aqsa), veröffentlicht in der digitalen Sammlung (Allgemeine Sammlung), Rn. $75 \mathrm{f}$.

24 EuG (Fn. 15), Rn. 106, 115, 120, 124; dies hat das EuG für das Home Secretary bereits in der Rs. Volksmudschaheddin des Iran II festgestellt, vgl. EuG (Fn. 12), Rn. $144 \mathrm{f}$.

25 Die Frage kam bereits in der Rs. Sison im Hinblick auf amerikanische Behördeninformationen auf, jedoch wollte der Rat sich im Rahmen seiner Begründung vor dem EuG nicht mehr ausdrücklich auf die amerikanischen Erkenntnisse stützen, vgl. EuG (Fn. 13), Rn. 64, 102.

$26 \operatorname{EuG}$ (Fn. 15), Rn. 129 ff.

27 Zur Abgrenzung der Schutzbereiche H. D. Jarass, Charta der Grundrechte der Europäischen Union, 2013, Art. 41 Rn. 17, Art. 47 Rn. 1, 5 sowie Art. 48 Rn. 16 f. betreffend Strafverfahren und Überschneidungen zu den allgemeineren Rechten.

28 EuG (Fn. 15), Rn. 138 f. sowie 146. 
laut EuG nicht heilen. ${ }^{29}$ Darüber hinaus gab es in praktischer Hinsicht Anhaltspunkte für in Indien stattfindende willkürliche Inhaftierungen, Folterungen und sogar außergerichtliche Hinrichtungen auf Grund von Terrorismusbekämpfungsvorschriften. ${ }^{30}$ Somit hat der zweite Klagegrund lediglich hinsichtlich der Drittstaatskooperation in concreto Erfolg.

Der dritte Klagegrund erweist sich aus institutioneller Perspektive und hinsichtlich der gerichtlichen Kontrolldichte der Ratsentscheidungen als aufschlussreich. Das EuG wiederholt die bisherige Rechtsprechung, nach der der Rat sich in seiner Begründung nicht in stereotypen Formulierungen verlieren darf, sondern die besonderen und konkreten Gründe angeben muss, aufgrund derer er in Ausübung seines Ermessens annimmt, dass der Betroffene auf die Liste gesetzt wird oder auf ihr verbleiben soll. Nur so wird eine Verteidigung des Betroffenen und damit die Wahrung seiner Verteidigungsrechte überhaupt möglich. Dabei dürfen die Gründe nicht aus der Luft gegriffen sein, sondern müssen sich auf nationale Beschlüsse zuständiger Behörden zurückführen lassen. ${ }^{31}$ Diese sekundärrechtliche Restriktion ist darauf zurückzuführen, dass der Rat über keinen eigenen Behördenunterbau zur Durchführung eigener Ermittlungen verfügt. ${ }^{32}$ Das EuG attestiert dem Rat zwar einen weiten Ermessensspielraum, behält sich aber eine Ermessensfehlerprüfung dergestalt vor, dass es prüft, ob die vom Rat angeführten Beweise alle zur Beurteilung relevanten Daten darstellen und die gezogenen Schlüsse zu stützen vermögen. Darüber hinaus prüft es die sachliche Richtigkeit, Zuverlässigkeit und Kohärenz der angeführten Beweise. ${ }^{33}$ Diese Feststellung des Umfangs gerichtlicher Kontrolldichte ist ebenso wenig neu wie die - zur Verteidigung des Rates - bislang fehlende gerichtliche Präzisierung derselben. ${ }^{34}$ Zumindest für das vorliegende Verfahren wäre dies aber ohnehin nicht weiterführend gewesen. Denn vor dem EuG stellt sich heraus, dass der Rat seine tragenden Begründungserwägungen auf eine Liste terroristischer Aktivitäten der Klägerin aus den Jahren 2005-2009 stützte, die jedoch in den ebenfalls angeführten Behördenbeschlüssen keine Erwähnung finden, da diese zum Großteil nur bis 2004 datieren. Der Rat führt also letztlich nicht auf Informationen zuständiger Behörden beruhende Erkenntnisse zur Begründung seiner Ermessensentscheidung an ${ }^{35}$ - woher stammen sie dann? Der Rat belegt seine zur Begründung angeführten „Hintergrundinformationen" schließlich mit aus dem Internet beschafften Presseartikeln und offenbart so ein eklatantes Verfehlen der Vorgaben des Gemeinsamen Standpunkts 931/2001/GASP: Er geht umgekehrt vor und stuft die Klägerin von Beginn an als Terroristin ein, anstatt sein Ermessen mit Hilfe nationaler Behördeninformation

$29 \operatorname{EuG}$ (Fn. 15), Rn. 141, 146 f., 149.

30 EuG (Fn. 15), Rn. 150.

31 EuG (Fn. 15), Rn. 161 f.; vgl. bereits zuvor das EuG in den Rs. Volksmudschaheddin des Iran I (Fn. 11), Rn. 143; Rs. Sison (Fn. 13), Rn. 60 sowie den EuGH in der Rs. Al-Aqsa (Fn. 13), Rn. 68 ff.

32 Vgl. insoweit EuGH, Rs. Al-Aqsa (Fn. 13), Rn. 69.

33 EuG (Fn. 15), Rn. 163, 220.

34 Vgl. EuG, Rs. Volksmudschaheddin des Iran II (Fn. 12), Rn. 55 m.w.N. zur Rechtsprechung.

35 EuG (Fn. 15), Rn. 168 f., 173 f. 
auszuüben und eine begründete Entscheidung zu treffen. ${ }^{36}$ Damit schwingt er sich selbst zur zuständigen Behörde im Sinne des Standpunkts auf und missachtet das mit dem Standpunkt etablierte zweistufige Informations- und Entscheidungssystem, ohne über eigene Ermittlungsressourcen zu verfügen. ${ }^{37}$ Wie kam der Rat zu diesem Verhalten? Dem Urteil lässt sich entnehmen, dass der Rat einer falschen Lesart der Handlungsgrundlagen erlag: er ging davon aus, sich bei einer Entscheidung für den Listenverbleib im Rahmen der regelmäßigen Überprüfung nur auf neue terroristische Aktivitäten stützen zu können. Jedoch darf er Betroffene auch auf Grundlage alter Beschlüsse auf der Liste belassen, soweit die Umstände dies rechtfertigen. Fehlende neue Behördenbeschlüsse rechtfertigen damit keine aus anderen Quellen angeführten „Hintergrundinformationen“.38 Der dritte Klagegrund hat damit vollen Erfolg.

\section{Bewertung und Ausblick}

Dass das EuG sich angesichts des Umstands, dass sich die Staatengemeinschaft schon nicht auf eine Definition von Terrorismus verständigen kann, grundsätzlich nicht auf eine Anwendungsbegrenzung der Vorschriften zur Terrorismusbekämpfung durch humanitäres Völkerrecht einlassen würde, überrascht nicht und wurde mit dem Verweis auf die Autonomie des Unionsrechts nachvollziehbar begründet. Die Abgrenzung zwischen unrechtmäßigen Kriegshandlungen im Sinne des humanitären Völkerrechts und terroristischen Handlungen dürfte auch nicht immer gelingen, Rechtsunsicherheit wäre die Folge. Der politische Charakter des besprochenen Falles und ähnlicher Verfahren, aus dem sich das vom EuG grundsätzlich anerkannte weite Ermessen des Rates erklärt, führt dazu, dass es sich konsequent auf eine Stärkung und Kontrolle verfahrensrechtlicher Aspekte beschränkt. Zur Prüfung der materiellen Frage nach dem terroristischen Charakter der Organisation kommt es nicht, ${ }^{39}$ die Konzentration liegt aus richterlicher Perspektive aus guten Gründen auf der formellen Prüfung der Wahrung nicht abdingbarer Verfahrensgrundrechte auf dem Weg zu einer solchen materiellen Entscheidung.

Die Entscheidung bringt hinsichtlich des Kreises der zuständigen Behörden Bekanntes, mit der Feststellung der Zulässigkeit drittstaatlicher Behördenkooperation angesichts der globalen Kooperationsbedürftigkeit in der Sache aber auch eine erfreuliche Klarstellung. Mögliche Gefahren für die Verteidigungsrechte der Be-

36 EuG (Fn. 15), Rn. 184, 186, 189, besonders deutlich Rn. 191 f.; am 17. Dezember 2014 erging das Urteil des EuG in der Rs. T-400/10 (Hamas/Rat der EU), noch nicht in der Sammlung veröffentlicht, in dem der Rat sich ebenfalls auf Informationen aus dem Internet und der Presse stützte, vgl. dort Rn. 109 ff., 121.

$37 \mathrm{EuG}$ (Fn. 15), Rn. 198, 203; jenseits kompetenziellen Denkens dürfte die Internetrecherche in keiner mitgliedstaatlichen Ermittlungsbehörde eine anerkannte oder ausreichende Ermittlungsmethode sein.

38 EuG (Fn. 15), Rn. 209, 212, 215; dieses Missverständnis trieb den Rat so weit, den für eine stichhaltige Begründung fehlenden Verweis auf Beschlüsse zuständiger Behörden der Klägerin zuzurechnen, da diese es versäumt habe, gegen sie auf nationaler Ebene ergriffene restriktive Maßnahmen gerichtlich vorzugehen und somit brauchbare „neue“ Behördeninformationen gleichsam zu produzieren, vgl. a.a.O., Rn. 205, $207 \mathrm{f}$.

39 Das Gericht selbst betont diesen Umstand, vgl. EuG (Fn. 15), Rn. 226. 
troffenen im Rahmen der Kooperation mit Drittstaaten sind im Schrifttum schon länger ausgemacht. ${ }^{40}$ Den Bedenken begegnet das EuG mit einer nicht näher spezifizierten Prüfpflicht des Rates hinsichtlich der rechtsstaatlichen Lage im betreffenden Drittland. Hier wären im Interesse des Rates noch genauere Angaben zur Prüfpflicht angezeigt gewesen, das Gericht nähert sich diesen aber lieber tastendnegativ. Dass der pauschale Verweis auf fremdes Recht nicht ausreicht, leuchtet ein - doch worauf kann der Rat sich bei seiner Prüfung stützen, wenn ihm, wie festgestellt, schon eigene Ermittlungsressourcen fehlen? Genügen Berichte von UN-Einrichtungen wie dem Menschenrechtsrat, dem UNHCR, bedarf es positiver Einschätzungen von EU-Institutionen, dem Europarat oder darf der Rat sich hierfür nur auf mitgliedstaatliche Informationen verlassen? Das dezentrale Informations- und Entscheidungssystem ist institutionell jedenfalls einer eigenen, dem Rat unterstehenden Ermittlungsbehörde vorzuziehen - so können Betroffene sowohl den nationalen Rechtsweg unmittelbar gegen etwaige Behördenentscheidungen beschreiten, als auch den unionsrechtlichen, um die Ratsentscheidung verfahrensrechtlich prüfen zu lassen.

Die politische Dimension des Verfahrens wird anhand der Presseerklärung des Rates deutlich, in der die Einlegung eines Rechtsmittels angekündigt und betont wird, es handle sich um eine rein verfahrensrechtlich-juristische, keine inhaltlichpolitische Entscheidung über die Organisation. ${ }^{41}$ Damit ist die Wirkung der Nichtigerklärung des EuG bis zu einer Entscheidung des EuGH suspendiert und diesem Gelegenheit gegeben, die Prüfpflichten des Rates im Rahmen der Informationsbeschaffung aus Drittstaaten zu präzisieren. ${ }^{42}$ Gegen das kurze Zeit später ergangene Urteil des EuG betreffend die Listung der Hamas, dem vergleichbare prozedurale Umstände zugrunde lagen, hat der Rat ebenfalls Rechtsmittel zum EuGH eingelegt. ${ }^{43}$ Dies verdeutlicht den ausstehenden verfahrensrechtlichen Klärungsbedarf im Kontext der vom Rat verwalteten Sanktionsliste.

40 Vgl. nur E. Spaventa, Fundamental What? The Difficult Relationship between Foreign Policy and Fundamental Rights, in: Cremona/de Witte (Eds.), EU Foreign Relations Law: Constitutional Fundamentals, 2008, S. 233, 246 ff.; dies., Fundamental rights and the interface between second and third pillar, in: Dashwood/ Maresceau (Eds.), Law and practice of EU External Relations: salient features of a changing landscape, 2008, S. 129, $141 \mathrm{ff}$.

41 Vgl. die Presseerklärung der Europäischen Union vom 2. Dezember 2014, abrufbar unter: http://eeas.europa.e u/delegations/sri_lanka/documents/press_corner/20141202_01.pdf (Stand: 1. März 2015).

42 Zur Suspendierung vgl. Art. 60 UAbs. 2 EuGH-Satzung; das Verfahren ist als Rs. C-599/14 P anhängig.

43 Vgl. Fn. 36; das Verfahren ist als Rs. C-79/15 P beim EuGH anhängig. 